\title{
Electromagnetic form factors of $\Lambda$ hyperon in the vector meson dominance model
}

\author{
Yongliang Yang, Dian-Yong Chen $\odot{ }^{*}$ and Zhun $\mathrm{Lu}^{\dagger}$ \\ School of Physics, Southeast University, Nanjing 211189, China
}

(Received 4 February 2019; published 17 October 2019)

\begin{abstract}
We perform an analysis on the electromagnetic form factors of $\Lambda$ hyperon in the timelike reaction $e^{+} e^{-} \rightarrow \Lambda \bar{\Lambda}$ by using a modified vector meson dominance model. We consider both the intrinsic structure components and the meson clouds components. For the latter one, we not only include the contributions from the $\phi$ and $\omega$ mesons, but also take into account the contributions from the resonance states $\omega(1420)$, $\omega(1650), \phi(1680)$, and $\phi(2170)$. We extract the model parameters by combined fit to the timelike effective form factor $\left|G_{\text {eff }}\right|$, the electromagnetic form factor ratio $\left|G_{E} / G_{M}\right|$ of $\Lambda$ hyperon from BABAR and BESIII Collaborations. We find that the vector meson dominance model can simultaneously describe these observables. Particularly, the inclusion of the resonance states in the model is necessary for explaining the ratio $\left|G_{E} / G_{M}\right|$ in a wide range of $\sqrt{s}$ as well as the form factor ratio. With the fitted parameter, we predict the relative phase $\Delta \Phi$, which is consistent with the recent measurement from BES III Collaboration. Moreover, we predict the single and double polarization observables, which could be measured in polarized annihilation reactions. We also analytically continue the expression of the form factors to spacelike region and estimate the spacelike form factors of $\Lambda$ hyperon.
\end{abstract}

DOI: 10.1103/PhysRevD.100.073007

\section{INTRODUCTION}

The electromagnetic form factors (EMFFs) $G_{E}$ and $G_{M}$ of hadrons are fundamental quantities for probing the internal structure of hadrons and understanding the perturbative and nonperturbative quantum chromodynamics (QCD) effects encoded in hadrons [1-4]. They contain the information on the distribution of electric charge and magnetic moment of the hadron. In the past decades the experimental and theoretical investigations on nucleon (proton and neutron) EMFFs [3-23], particularly the effective form factor, have been performed in both the spacelike and timelike regions, e.g., in the $e p$ elastic scattering, $\bar{p} p$ annihilation or $e^{+} e^{-}$annihilation processes. A reasonable theoretical approach to understand the nucleon EMFFs in the spacelike region is the vector meson dominance (VMD) model, which has been extended to study the timelike data [5,6,24-27]. In the framework of the VMD model, the EMFFs can be naturally expressed as the product of the two components: an intrinsic structure (the valance quark) and the meson cloud ( $q \bar{q}$ pairs). Other

\footnotetext{
*hendy@seu.edu.cn

tzhunlu@seu.edu.cn
}

Published by the American Physical Society under the terms of the Creative Commons Attribution 4.0 International license. Further distribution of this work must maintain attribution to the author(s) and the published article's title, journal citation, and DOI. Funded by SCOAP. than the VMD model, the pQCD inspired model has also been applied to parametrize the nucleon EMFFs $[14,17,28-30]$, which have an analytical form that can also be extended to the timelike region.

In recent years, there is also increasing interest on the EMFFs of $\Lambda$ hyperon from both the theoretical [31-38] and experimental side $[8,39,40]$. In contrast to the nucleon, it is rather difficult to explore the scattering cross section and the EMFFs of $\Lambda$ hyperon in the spacelike region [8,39-41], since the hyperons are unstable and hyperon targets are unfeasible. The EMFFs of hyperons in the spacelike region are hardly to be measured by exclusive experiments, therefore, the timelike form factors in reaction $e^{+} e^{-} \rightarrow$ $\Lambda \bar{\Lambda}$ can offer a unique opportunity to study the electromagnetic property of $\Lambda$ hyperon. Experimentally, the cross sections for the $e^{+} e^{-} \rightarrow \Lambda \bar{\Lambda}$ have been measured by $B A B A R$ and BESIII Collaborations [39,40,42], a notable feature of the extracted effective form factor $G_{\text {eff }}$ is the near threshold enhancement. Similar enhancement effect can also be observed in the timelike effective form factor of proton $[15,18,20,22]$ and such phenomena can be interpreted by the Coulomb final-state interactions [43,44], since the proton is a charged particle. As for $\Lambda$ hyperon, $\Lambda$ hyperon is neutral, thus, such kind of final-state interactions vanish. Then decoding the source of the near threshold of enhancement in the $\Lambda$ hyperon effective form factor will be a intriguing question. Moreover, $G_{E}$ and $G_{M}$ in the timelike region are complex, there is a 
relative phase angle $\Delta \Phi$ between these two form factors. During preparation the present work, a preliminary measurement [45] from the BESIII Collaboration demonstrates a rather large phase $\Delta \Phi=42^{\circ} \pm 16^{\circ} \pm 8^{\circ}$ at $\sqrt{s}=2.396 \mathrm{GeV}$. However, there is very little theoretical implication on the relative phase $\Delta \Phi$ other than the $\Lambda \Lambda$ potential model [31].

In the present work, we aim at simultaneously describing the available data on the effective form factor $\left|G_{\text {eff }}\right|$ and the ratio $\left|G_{E} / G_{M}\right|$ from both BABAR and BESIII Collaborations in light of the VMD model. Due to the isoscalar property of the $\Lambda$, the contribution of $\rho$ meson and its resonances should be excluded. Furthermore, the threshold of $\Lambda \bar{\Lambda}$ is about $2231 \mathrm{MeV}$, thus $\omega$ and $\phi$, as well as their resonances below the threshold should be involved. In the present work, we take into account the contributions from the resonance states $\omega(1420)$, $\omega(1650), \phi(1680)$, and $\phi(2170)$. The formula of timelike form factors are obtained by an analytic continuation of the spacelike form factors, and in the timelike region, we take into account the decay widths of the vector mesons and their resonance states in order to introduce a complex structure for $G_{E}$ and $G_{M}$ [43].

The work is organized as follows: an analysis of $\Lambda$ hyperon form factors in the VMD model is performed after the Introduction. In Sec. III, we present our fit to the timelike form factor of $\Lambda$ hyperon and our predictions for the single and double polarization observables as well as spacelike form factors and Sec. IV is devoted to a short summary.

\section{ANALYSIS OF FORM FACTORS OF $\Lambda$ HYPERON IN THE VMD MODEL}

Encouraged by the success of the VMD model for nucleon (proton and neutron) in Ref. [27], we extend the model to investigate the EMFFs of the $\Lambda$ hyperon. First, we introduce the $\Lambda$ form factors in the spacelike region, where $q^{2}=-Q^{2}<0$. Considering the relativistic invariance of the EMFFs, one can write down the electromagnetic current of a baryon with spin-1/2 in terms of the Dirac form factor $F_{1}\left(Q^{2}\right)$ and Pauli form factor $F_{2}\left(Q^{2}\right)$ as

$$
J^{\mu}=\gamma^{\mu} F_{1}\left(Q^{2}\right)+\frac{i \sigma^{\mu \nu} q_{\nu}}{2 m_{\Lambda}} F_{2}\left(Q^{2}\right) .
$$

In the VMD model, $F_{i}$ can be further decomposed as

$$
F_{i}=F_{i}^{S}+F_{i}^{V}
$$

where $F_{i}^{S}$ and $F_{i}^{V}$ denote the isoscalar and isovector components of the form factors, respectively. As for $\Lambda$ hyperon, there is only isoscalar contribution, i.e., $F_{i}^{V} \equiv 0$, since $\Lambda$ hyperon is a isospin singlet.
Based on the above consideration, the observed EMFFs of $\Lambda$ hyperon can be expressed in terms of the $F_{i}^{S}$ by,

$$
G_{M}=F_{1}^{S}+F_{2}^{S}, \quad G_{E}=F_{1}^{S}-\tau F_{2}^{S},
$$

where $\tau=Q^{2} / 4 m_{\Lambda}^{2}$. We note that these relations satisfy the kinematical constraint $G_{E}\left(-4 m_{\Lambda}^{2}\right)=G_{M}\left(-4 m_{\Lambda}^{2}\right)$. This constraint is important in the timelike region [26].

In the VMD models for the nucleon $[5,6,25,27]$, the form factors are attributed to two parts. One is the intrinsic structure which is determined by the valence quarks, the other is the meson cloud. The nature of the intrinsic structure has been discussed in details in Refs. [6,25]. In those studies the form factors satisfy the asymptotic behavior of pQCD $[30,46]$. While, the meson cloud term was used to describe the interaction between the bare nucleon and the photon in the framework of the vector meson dominance $(\rho, \omega$ and $\phi)[5,26]$.

\section{A. Spacelike form factors}

In light of the VMD, we consider the isoscalar components of the form factors which receive contributions from the $\omega$ and $\phi$ meson. The Dirac and Pauli form factors of $\Lambda$ hyperon are expressed as the product of the intrinsic form factor $g\left(Q^{2}\right)$ and the terms from VMD. In addition, we will also consider the contributions from the resonance states of $\phi$ and $\omega$, which are $\omega(1420), \omega(1650), \phi(1680)$, and $\phi(2170)$. The expressions of the form factors from these resonance states are assumed to be the same as those from the vector meson $\omega(782)$ and $\phi(1020)$ in our modified model. The Dirac and Pauli form factors of $\Lambda$ hyperon are normalized at $Q^{2}=0$ as,

$$
F_{1}(0)=0, \quad F_{2}(0)=G_{M}(0)=\mu_{\Lambda} .
$$

It should be noticed the magnetic moment of $\Lambda$ hyperon is the origin of the magnetic form factor, which can be in unit of baryon natural magneton, i.e., $\hat{\mu}_{\Lambda} \equiv e /\left(2 m_{\Lambda}\right)$, or nucleon magneton, i.e., $\hat{\mu}_{N} \equiv e /\left(2 m_{N}\right)$. The experimental measurement indicate $\mu_{\Lambda}=-0.613 \hat{\mu}_{N}=-0.723 \hat{\mu}_{\Lambda}$ [47]. Here and after, we will use the baryon neutral magneton as the unit of electromagnetic momentum if there is no special declaration.

For large values of $Q^{2}$, the development of $\mathrm{pQCD}$ has put constraints to the asymptotic behavior of form factors, $F_{1} \sim 1 / Q^{4}$ and $F_{2} \sim 1 / Q^{6}[5,28,29]$. These constraints lead to the parametrized forms of the isoscalar Dirac and Pauli form factors in the VMD model as follows,

$$
\begin{aligned}
F_{1}^{S}\left(Q^{2}\right)= & \frac{g\left(Q^{2}\right)}{3} \Sigma_{i=1}^{N}\left[-\beta_{\omega_{i}}-\beta_{\phi_{i}}+\beta_{\omega_{i}} \frac{m_{\omega_{i}}^{2}}{m_{\omega_{i}}^{2}+Q^{2}}\right. \\
& \left.+\beta_{\phi_{i}} \frac{m_{\phi_{i}}^{2}}{m_{\phi_{i}}^{2}+Q^{2}}\right]
\end{aligned}
$$




$$
\begin{aligned}
F_{2}^{S}\left(Q^{2}\right)= & \frac{g\left(Q^{2}\right)}{3} \Sigma_{i=1}^{N}\left[\left(\mu_{\Lambda}-\alpha_{\phi_{i}}\right) \frac{m_{\omega_{i}}^{2}}{m_{\omega_{i}}^{2}+Q^{2}}\right. \\
& \left.+\alpha_{\phi_{i}} \frac{m_{\phi_{i}}^{2}}{m_{\phi_{i}}^{2}+Q^{2}}\right]
\end{aligned}
$$

where $N=3$ and $\mu_{\Lambda}=-0.723 \hat{\mu}_{\Lambda} . \omega_{i}(i=1,2,3)$ denotes the vector meson states $\omega(782), \omega(1420)$, and $\omega(1650)$, and $\phi_{i}(i=1,2,3)$ denotes the vector meson states $\phi(1020), \phi(1680)$, and $\phi(2170)$. In the present VMD scenario, the virtual photon couples to the hyperon via vector mesons. It should be noted that there exist the mechanism where the virtual photon couples to the pseudoscalar meson cloud surrounding the baryon as discussed in Refs. [48-51]. Such kind of mechanism is not included in the present work due to insufficient experimental data of $\Lambda$ hyperon form factors.

In the VMD model, the intrinsic structure factor is characterized by a three-valence-quark [6] and is chosen in a dipole form $g\left(Q^{2}\right)=\left(1+\gamma Q^{2}\right)^{-2}$, which enables a good fit to the nucleon EMFFs [25,27]. With the particular expression of $g\left(Q^{2}\right)$, one can find for the large value of $Q^{2}$, $F_{1} \sim g\left(Q^{2}\right) \sim 1 / Q^{4}$ and $F_{2} \sim g\left(Q^{2}\right) / Q^{2} \sim 1 / Q^{6}$, which is consistent with the asymptotic behavior of $F_{1}$ and $F_{2}$ estimated by leading order of pQCD [5,28,29].

In the present scenario, the parameter $\gamma$ in $g\left(Q^{2}\right)$ and the coefficients $\beta_{\omega_{i}}, \beta_{\phi_{i}}, \alpha_{\phi_{i}}$ in Eqs. (5)-(6) are free parameters, which can be fitted by the data of timelike electromagnetic form factors. These coefficients can be explained as the products of a $V \gamma$ coupling constant and $V B B$ coupling constant [27]. The VMD model is valid in both spacelike and timelike region, the parameters in both regions are usually considered to be unified, thus these parameters should be real since the form factors in the spacelike region are real. In the present work, our main purpose is to investigate the existing data for timelike form factors, we do not further elaborate the spacelike expressions. We will extend the expressions of the form factor to the timelike region in the next subsection.

\section{B. Timelike form factors}

The general expression of the Born cross section for the reaction of $e^{+} e^{-} \rightarrow \bar{B} B$ has been given in Ref. [21] under the one-photon exchange approximation with $B$ as a spin- $\frac{1}{2}$ baryon. The integrated cross section of the $\Lambda$ hyperon pairs production is governed by the electric and magnetic form factors $G_{E}$ and $G_{M}$ as,

$$
\sigma(s)=\frac{4 \pi \alpha^{2} \beta}{3 s} C_{\Lambda}\left[\left|G_{M}(s)\right|^{2}+\frac{1}{2 \tau}\left|G_{E}(s)\right|^{2}\right]
$$

where $G_{E}(s)$ and $G_{M}(s)$ are the EMFFs in the timelike region, $s$ is the square of the center of mass (c.m.) energy, $\tau=s / 4 M_{\Lambda}^{2}$. The variable $\beta=\sqrt{1-1 / \tau}$ is a phase-space factor. The Coulomb enhancement factor $C_{\Lambda}$ [38,52], accounting for the electromagnetic interaction of the pointlike baryon pairs in the final state, is $C_{\Lambda}=1$ for a neutral baryon pair. Another quantity used in various analyses is the effective form factor $G_{\text {eff }}(s)$. The effective form factor is equivalent to $\left|G_{M}\right|$ under the hypothesis $G_{E}=G_{M}$ [17]. In general cases, the effective form factor can be expressed in terms of the moduli of the EMFFs [15]:

$$
\begin{aligned}
\left|G_{\mathrm{eff}}(s)\right| & =\sqrt{\frac{2 \tau\left|G_{M}(s)\right|^{2}+\left|G_{E}(s)\right|^{2}}{1+2 \tau}} \\
& =\sqrt{\frac{\sigma_{e^{+} e^{-} \rightarrow \bar{\Lambda} \Lambda}(s)}{\frac{4 \pi \alpha^{2} \beta}{3 s} C_{\Lambda}\left[1+\frac{1}{2 \tau}\right]}} .
\end{aligned}
$$

It is proportional to the square root of the Born cross section, which can be extracted from experimental measurements on the cross section for $e^{+} e^{-} \rightarrow \Lambda \bar{\Lambda}$.

By an appropriate analytic continuation in the complex plane, the form factors of Eqs. (5)-(6) can be used to analyze the form factors in the timelike region $[5,17]$. For the intrinsic structure, the analytical continuation to the timelike region is based on the following relation [17]:

$$
Q^{2}=-q^{2}=q^{2} e^{i \pi}
$$

Therefore, in the timelike region, $g\left(q^{2}\right)$ has the form of an analytical continuation form:

$$
g\left(q^{2}\right)=\frac{1}{\left(1-\gamma q^{2}\right)^{2}} .
$$

It should be noticed that $\gamma$ is a positive parameter, thus $g\left(q^{2}\right)$ will have a pole in the position $q^{2}=1 / \gamma$, such pole could be removed from the real axes by changing the relations in Eq. (9) with $Q^{2} \rightarrow q^{2} e^{i \theta}$, where $\theta \neq \pi$. In such scenario, the analytical continuation of the form factor will be destroyed in a certain extend. To evade this problem, one can restrict the pole in the unphysical region of the form factors, which indicate $\gamma$ should satisfy $\gamma>1 /\left(4 m_{\Lambda}^{2}\right)$ for $\Lambda$ form factor.

For the meson cloud part, we take into account the width of vector mesons [43] to introduce the complex structure in the timelike region. By the following replacement,

$$
\begin{gathered}
\beta_{\omega_{i}} \frac{m_{\omega_{i}}^{2}}{m_{\omega_{i}}^{2}+Q^{2}} \rightarrow \beta_{\omega_{i}} \frac{m_{\omega_{i}}^{2}}{m_{\omega_{i}}^{2}-q^{2}-i m_{\omega_{i}} \Gamma_{\omega_{i}}}, \\
\beta_{\phi_{i}} \frac{m_{\phi_{i}}^{2}}{m_{\phi_{i}}^{2}+Q^{2}} \rightarrow \beta_{\phi_{i}} \frac{m_{\phi_{i}}^{2}}{m_{\phi_{i}}^{2}-q^{2}-i m_{\phi_{i}} \Gamma_{\phi_{i}}},
\end{gathered}
$$

we can obtain the modified VMD model in the timelike region. It should be noticed that in the case of nucleon, the timelike form factors have been analyzed in a VMD 
model $[5,6]$, where only the ground vector mesons were included. It was found that the complex structure of nucleon timelike form factors came from a undetermined phase angle $\theta$ in $g\left(q^{2}\right)$ as well as the width of $\rho$ meson [53]. In the present model, we include the excited states of the vector meson states and the widths of involved states are crucial for reproducing the relative phase angle $\Delta \Phi$, which is similar to the case of the pion form factor studied in Ref. [54] through the VMD model. In the present model, we do not consider the phase angle in intrinsic $g\left(q^{2}\right)$ to keep the validity of the fit. Moreover, the decay properties of these $\omega$ and $\phi$ resonances are not well determined experimentally. Thus, in the present work, we still use a constant decay width as approximation.

\section{NUMERICAL RESULTS AND DISCUSSIONS}

\section{A. Fit the timelike form factors}

The masses and widths of the involved vector mesons are listed in Table I, which are taken from the most recent experimental measurements from $B A B A R$ [55,56], CMD-3 [57], and BESIII [58] Collaborations. With the expressions of $F_{1}$ and $F_{2}$ in Eqs. (5)-(6) and the replacements in Eq. (11), we perform a combined fit to the effective form factor $G_{\text {eff }}$ in the region $2.2324 \mathrm{GeV}<\sqrt{s}<3.08 \mathrm{GeV}$, the electromagnetic form factor ratio. It should be notice that the newly measured relative phase has a large uncertainty [45], thus in the present work, we only fit the data of the effect form factor and form factor ratio [39,40,42]. With the fitted parameters, we can predict the relative phase. By comparing our prediction with the experimental from BES III [45], we can further check the reliability of the present fit. In the present scenario, there are 16 experimental data and 10 free parameters. The value of intrinsic parameter $\gamma$ is fitted to be $0.336 \mathrm{GeV}^{-2}$ and the other parameters are summarized in Table II. It should be noticed that $g\left(q^{2}\right)$ has a pole in the position $q^{2}=1 / \gamma$, corresponding $q^{2}=$ $2.976 \mathrm{GeV}^{2}$ or $q=1.725 \mathrm{GeV}$. Since that pole is below

TABLE I. The masses and widths of the involved vector mesons in unit of $\mathrm{MeV}$ [55-58].

\begin{tabular}{lrcccc}
\hline \hline State & Mass & Width & State & Mass & Width \\
\hline$\omega(782)[55]$ & 782 & 8.1 & $\phi(1020)[56]$ & 1019 & 4.2 \\
$\omega(1420)[57]$ & 1418 & 104 & $\phi(1680)[57]$ & 1674 & 165 \\
$\omega(1650)[57]$ & 1679 & 121 & $\phi(2170)[58]$ & 2171 & 128 \\
\hline \hline
\end{tabular}

TABLE II. The parameters obtained from the combined fit.

\begin{tabular}{lrcrcc}
\hline \hline Parameter & Value & Parameter & \multicolumn{1}{c}{ Value } & Parameter & \multicolumn{1}{c}{ Value } \\
\hline$\beta_{\omega(782)}$ & 1.248 & $\beta_{\omega(1420)}$ & 0.712 & $\beta_{\omega(1650)}$ & 1.0129 \\
$\beta_{\phi(1020)}$ & -1.902 & $\beta_{\phi(1680)}$ & -0.581 & $\beta_{\phi(2170)}$ & -0.584 \\
$\alpha_{\phi(1020)}$ & -2.224 & $\alpha_{\phi(1680)}$ & 2.748 & $\alpha_{\phi(2170)}$ & 0.615 \\
\hline \hline
\end{tabular}

the threshold and we are working above the threshold, we can ignore the effect of the pole in first approximation.

In Fig. 1, we present our fit to the $\Lambda$ effective form factor $\left|G_{\text {eff }}\right|$ in the energy range $2.2324 \mathrm{GeV}<\sqrt{s}<3.08 \mathrm{GeV}$. For comparison, we also present the theoretical estimations from the potential model up to $\sqrt{s}=2.4 \mathrm{GeV}$ [31]. After including the resonances of $\omega$ and $\phi$ mesons, the fit demonstrates that our model can accurately describe the effective form factor $\left|G_{\text {eff }}\right|$ of the $\Lambda$ hyperon. Additionally, the new BESIII data [45] is plotted as a star in Fig. 1, which is also consistent with our fit curve. In particular, we also present the intrinsic and meson cloud contributions in Fig. 1. We find that the intrinsic contribution is much smaller than the meson cloud in our VMD model.

In Fig. 2, we present our result of the electromagnetic form factor ratio. This ratio is determined to be 1 at the threshold due to the kinematical restriction. With $\sqrt{s}$ increasing, this ratio increased at first and then decreased, which is similar to estimation of the $\Lambda \bar{\Lambda}$ potential model [31]. Our fitted results reach up its maximum $\sim 1.25$ at $\sqrt{s} \simeq 2.28 \mathrm{GeV}$, which is consistent with the estimation of $\Lambda \bar{\Lambda}$ potential model. It should be noticed that for large value of $Q^{2}$ the asymptotic behaviors of form factors satisfy,

$$
\begin{aligned}
F_{1} & \sim \frac{g\left(q^{2}\right)}{3} \sum_{i}^{N}\left(-\beta_{\omega_{i}}-\beta_{\phi_{i}}\right)-\tau F_{2} \\
& \sim-\frac{g\left(q^{2}\right)}{3} \sum_{i}^{N}\left(\left(\mu_{\Lambda}-\alpha_{\phi_{i}}\right) \frac{m_{\omega_{i}}^{2}}{4 m_{\Lambda}^{2}}+\alpha_{\phi_{i}} \frac{m_{\phi_{i}}^{2}}{4 m_{\Lambda}^{2}}\right),
\end{aligned}
$$

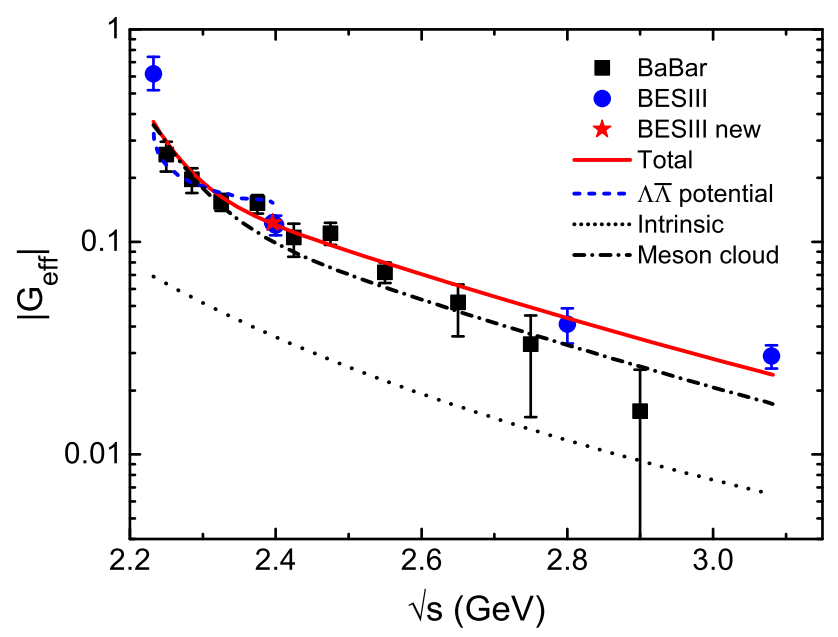

FIG. 1. Our fit to the effective form factor $\left|G_{\text {eff }}\right|$ of $\Lambda$ hyperon (solid curve). The black dotted and dashed-dotted curves refer to contributions from the intrinsic component and meson cloud, respectively. The rectangles, circles, and star represent the data from BABAR [39], BESIII [40,42] Collaborations and recent measurement from BESIII [45], respectively. For comparison, we also present the result from the $\Lambda \bar{\Lambda}$ potential model [31] (blue dashed curve). 


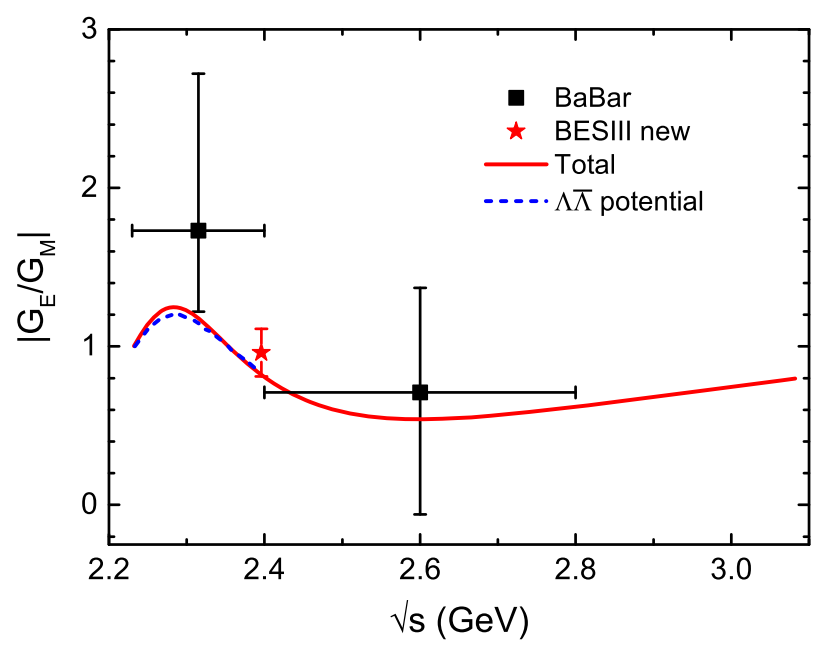

FIG. 2. The same as Fig. 1 but for the electromagnetic form factor ratio. The experimental data from $B A B A R$ Collaborations [39] are considered in the present fit, while the one from BESIII Collaboration [45] is presented for comparison. The red solid curve is our fit results. For comparison, we also present the result from the $\Lambda \bar{\Lambda}$ potential model [31] (blue dashed curve).

which indicate the form factor ratio is a constant in the limit of $q^{2} \rightarrow \infty$.

Our result of the relative phase $\Delta \Phi$ is presented in Fig. 3. We find that the new experimental measurement from BES III Collaboration [45] is consistent with our result (the red solid line). It should be noticed that $G_{E}=G_{M}$ on the $\Lambda \bar{\Lambda}$ threshold, thus, $\Delta \Phi=0$ on the threshold. In the combined fit, we find that the inclusion of the vector resonance states is crucial for describing the data on $\left|G_{\text {eff }}\right|$ and $\left|G_{E} / G_{M}\right|$. After including these vector resonances, the near threshold enhancements of effective form factor, the

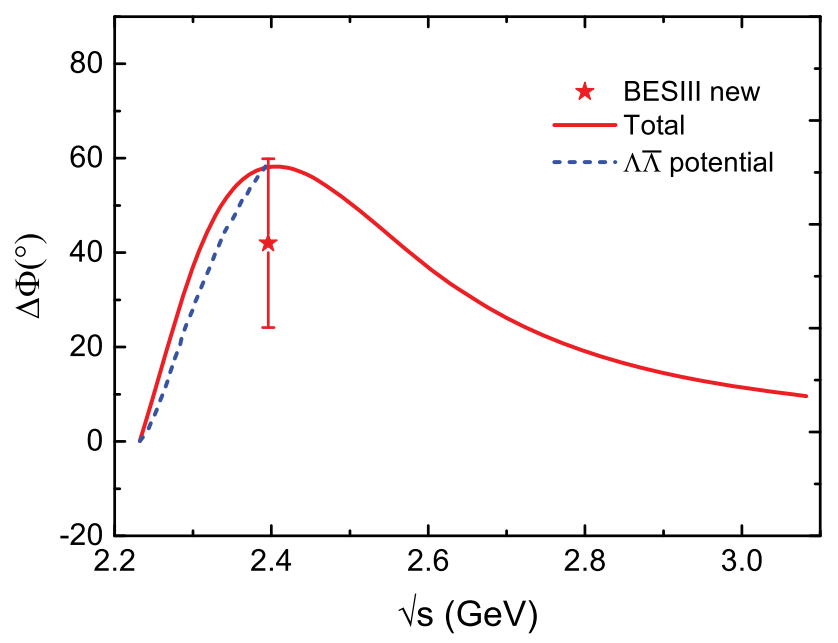

FIG. 3. The same as Fig. 1 but for the relative phase $\Delta \Phi$. The red star with error bar indicates the experimental data from BESIII Collaboration [45]. The red solid curve is the prediction of the present model. For comparison, we also present the result from the $\Lambda \bar{\Lambda}$ potential model [31] (blue dashed curve). large electromagnetic form factor ratio can be well reproduced. The widths of these resonances lead to a complex form factor in the timelike region and the relative phase angle also consistent with the experimental measurement, which further indicates the reliability of the present scenario.

\section{B. Polarization observables in timelike region}

With the fitted $G_{E}$ and $G_{M}$, we can estimate the spin polarized observables appearing in the reaction $e^{+} e^{-} \rightarrow$ $\bar{\Lambda} \Lambda[35,36]$. Under one-photon exchange approximation, the single and double polarization observables can be related to electromagnetic form factors by,

$$
\begin{aligned}
A_{y} & =\frac{-2 m_{\Lambda} \sqrt{s} \sin (2 \theta) \operatorname{Im}\left(G_{M} G_{E}^{*}\right)}{D_{c}-D_{s} \sin ^{2}(\theta)}, \\
A_{x z} & =\frac{2 m_{\Lambda} \sqrt{s} \sin (2 \theta) \operatorname{Re}\left(G_{M} G_{E}^{*}\right)}{D_{c}-D_{s} \sin ^{2}(\theta)}, \\
A_{x x} & =\frac{\left[D_{c}-D_{s}\right] \sin ^{2}(\theta)}{D_{c}-D_{s} \sin ^{2}(\theta)} \\
A_{y y} & =\frac{-D_{s} \sin ^{2}(\theta)}{D_{c}-D_{s} \sin ^{2}(\theta)} \\
A_{z z} & =\frac{\left[D_{s} \sin ^{2}(\theta)+D_{c} \cos ^{2}(\theta)\right]}{D_{c}-D_{s} \sin ^{2}(\theta)},
\end{aligned}
$$

where $D_{c}=2 s\left|G_{M}\right|^{2}, D_{s}=s\left|G_{M}\right|^{2}-4 m_{\Lambda}^{2}\left|G_{E}\right|^{2}$, and $\theta$ is the scattering angle defined in the c.m. frame. In this work, we present the polarized observables with $\theta=45^{\circ}$. From above expressions of the spin polarization observables, one can find that the observables $A_{x x}, A_{y y}$ and $A_{z z}$ depend on moduli of the EMFFs only. As for $A_{y}$ and $A_{x z}$, they not only depend on the moduli of the EMFFs, but also on the relative phase, since $G_{E} / G_{M}=\left|G_{E} / G_{M}\right| e^{i \Delta \Phi}$ [35].

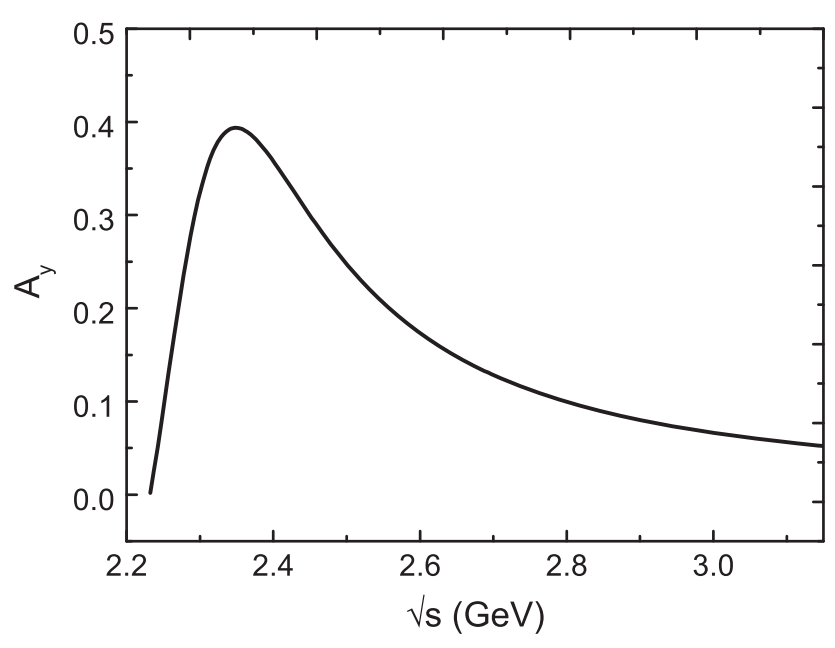

FIG. 4. Prediction for the single polarization observable $A_{y}$ vs $\sqrt{s}$ in $e^{+} e^{-} \rightarrow \Lambda \bar{\Lambda}$ at the fixed angle $\theta=45^{\circ}$. 

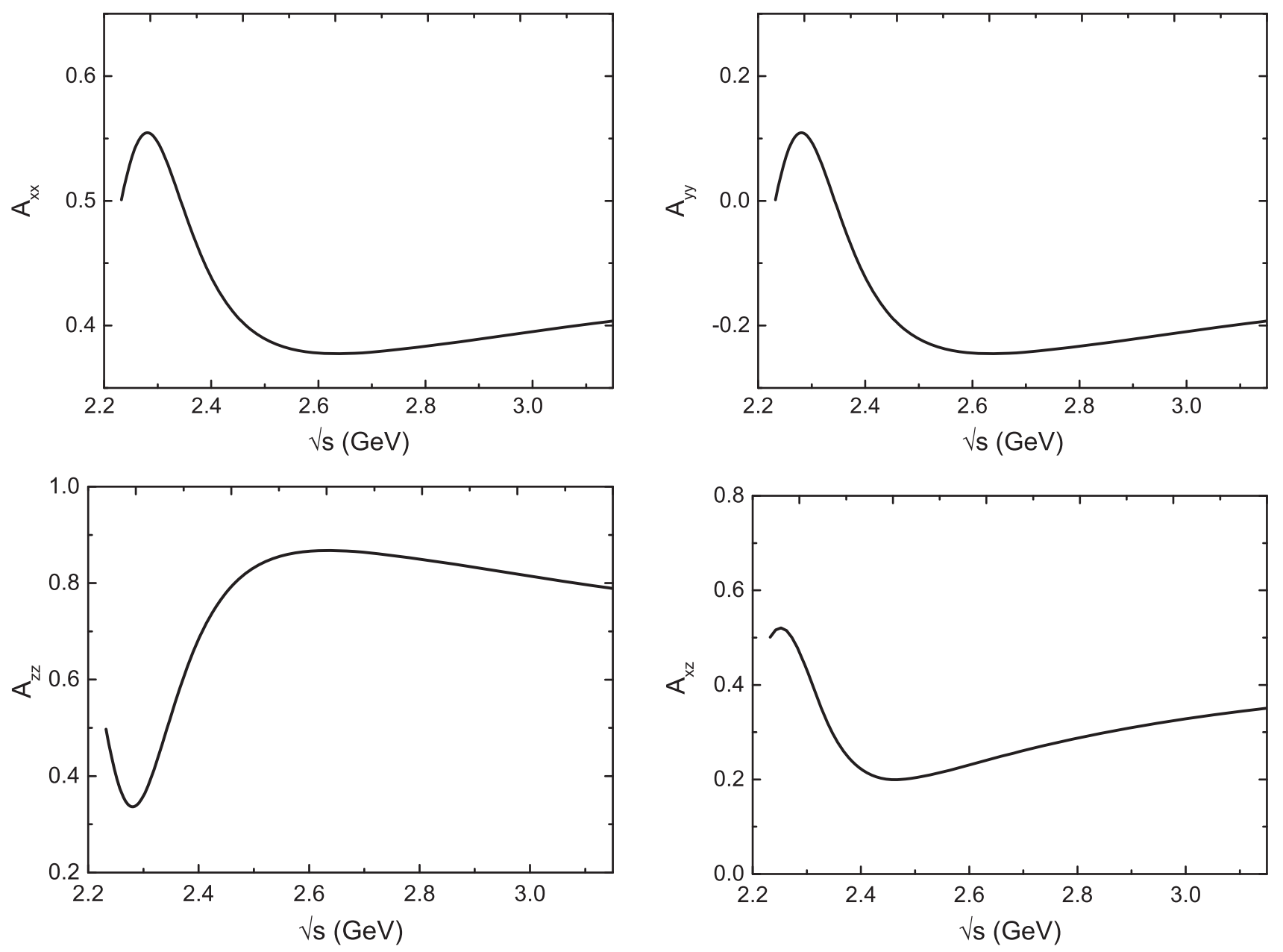

FIG. 5. Predictions for the double polarization observables $A_{x z}, A_{x x}, A_{y y}$ and $A_{z z}$ vs $\sqrt{s}$ in $e^{+} e^{-} \rightarrow \Lambda \bar{\Lambda}$ at the fixed angle $\theta=45^{\circ}$.

Therefore, precise measurements on these observables will be very useful for discriminating different models. In Fig. 4, we present the single polarization observable $A_{y}$ as function of $\sqrt{s}$. Since $\operatorname{Im}\left(G_{M} G_{E}^{*}\right) \sim \sin (\Delta \Phi)$, the shape of $A_{y}$ can be directly related to the relative phase $\Delta \Phi$, which indicates the precise measurement of the single spin polarization $A_{y}$ can provide a way to obtain the exact information of the relative phase $\Delta \Phi$. The double polarization observables depending on $\sqrt{s}$ are presented in Fig. 5.

\section{Form factors in spacelike region}

It is straightforward to extend our estimation to the spacelike region with the fitting parameters. Unlike the case of the nucleon, the $\Lambda$ hyperon spacelike EMFFs are hard to measure experimentally and no experimental data is available expect $\mu_{\Lambda}$ until now. But there are some theoretical investigations on EMFFs of $\Lambda$ hyperon in the spacelike region. In Ref. [59], EMFFs of $\Lambda$ hyperon in the spacelike region are estimated by using a combination of Dyson-Schwinger equation and Bethe-Salpeter equation, where the former equation is used to calculate the quark propagator and the latter one is adopted to describe the baryons as bound states of three valence quarks. The EMFFs were also estimated in a relativistic quark model, where both contributions from the quark core and meson cloud are considered [60]. The lattice QCD estimated the $\Lambda$ hyperon EMFFs at $Q^{2}=0.227(2) \mathrm{GeV}^{2}$ with unphysical pion mass [61].

With the fitted parameters in Table II, we also predict EMFFs in the spacelike region. It should be notice that the estimations in Refs. [59-61] are in unit of nucleon magneton $\hat{\mu}_{N}$. To compare our predictions with the estimation in previous literatures, we convert the unit of the results in Refs. [59-61] into natural baryon magneton. In Fig. 6, we present our numerical results of spacelike EMFFs and also show the estimations of $\Lambda$ hyperon EMFFs from different model [59,60] and lattice QCD [61] for comparison. Our estimation indicate the lineshape of $G_{E}$ has a zero crossing at $Q^{2}=0.25 \mathrm{GeV}^{2}$ and it becomes positive in the region of $Q^{2}>0.25 \mathrm{GeV}^{2}$. Such property is similar to the one estimated in Ref. [60], whose zero crossing is located around $1 \mathrm{GeV}^{2}$. While the estimation in Ref. [59] indicated that there are no zero crossing in the 

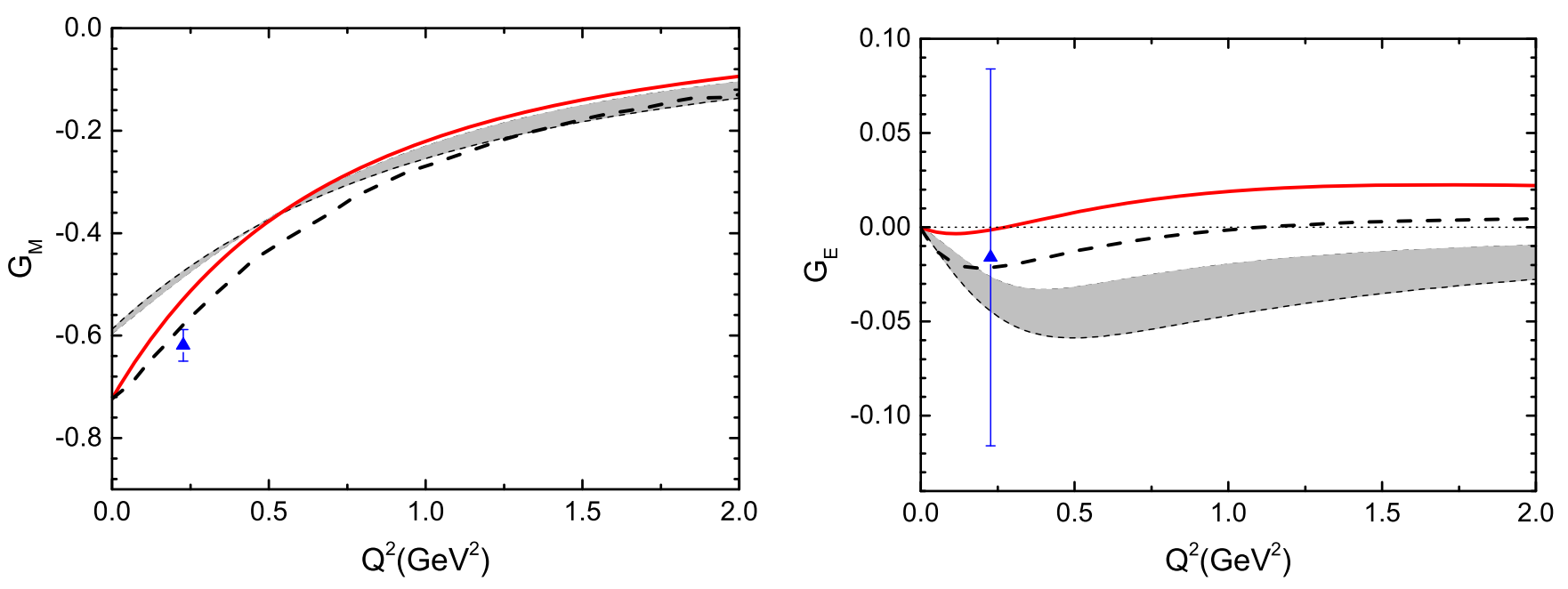

FIG. 6. The EMFFs of $\Lambda$ hyperon from the present estimation (solid lines) comparing with the predictions of Ref. [59] (shadow area), Ref. [60] (dashed lines) and the lattice data (triangles point with error bar) from Ref. [61].

estimated $Q^{2}$ range. As for the magnetic form factor, its magnitude and shape are consistent with other model predictions $[59,60]$.

With the electromagnetic form factors, one could estimate the magnetic and charge radius by,

$$
\left\langle r^{2}\right\rangle_{M}=-\left.6 \frac{1}{\mu_{\Lambda}} \frac{d G_{M}}{d Q^{2}}\right|_{Q^{2}=0}, \quad\left\langle r^{2}\right\rangle_{E}=-\left.6 \frac{d G_{E}}{d Q^{2}}\right|_{Q^{2}=0} .
$$

With the parameters in the present work, the magnetic and charge radius are estimated to be,

$r_{M}=\sqrt{\left\langle r^{2}\right\rangle_{M}}=0.42 \mathrm{fm}, \quad r_{E}=\sqrt{\left\langle r^{2}\right\rangle_{E}}=0.11 \mathrm{fm}$.

The estimated charge radius are consistent with the theoretical estimation in the chiral constitute quark model $[62,63]$, which are $0.131 \mathrm{fm}$. However, the estimated magnetic radius is much smaller than the one estimated in Refs. [62,63] and the PDG average of neutron magnetic radius [47], which are $0.801 \mathrm{fm}$ and $0.864_{-0.008}^{+0.009} \mathrm{fm}$, respectively.

\section{SUMMARY}

In the present work, we have presented an analysis on the EMFFs of the $\Lambda$ hyperon in a modified VMD model. We take into account the contributions of two components, which are the valence quarks and the meson cloud in the VMD model. Different from the case of the nucleon, the isovector parts of the Dirac and Pauli form factors vanish due to the isoscalar nature of the $\Lambda$ hyperon. We further analytically continue the EMFFs from the spacelike region to the timelike region.

To fit the timelike effective form factor $G_{\text {eff }}$ and electromagnetic form factor ratio $\left|G_{E} / G_{M}\right|$, we involve all the isospin singlet vector meson below the $\Lambda \bar{\Lambda}$ threshold, which are $\omega(782), \omega(1420), \omega(1650), \phi(1020), \phi(1680)$, and $\phi(2170)$. With these vector mesons, we can well reproduce the experimental data, and we also find the vector resonances, $\omega(1420), \omega(1650), \phi(1680)$, and $\phi(2170)$, are crucial in depicting the experimental data.

With the parameters obtained by the combined fit of $\left|G_{\text {eff }}\right|$ and $\left|G_{E} / G_{M}\right|$, we compared the preliminary results of $\left|G_{\text {eff }}\right|,\left|G_{E} / G_{M}\right|$ and $\Delta \Phi$ from BESIII Collaboration with our model, these new data are also consistent with our fit results. We predicted the single and double spin polarization observables of $e^{+} e^{-} \rightarrow \Lambda \bar{\Lambda}$ process, which could be tested by further precise measurements. Moreover, we analytically continue the expressions of the form factors from the timelike region to the spacelike region. Our estimations are qualitatively similar to other model calculations and lattice estimation but slight different quantitatively.

The estimations of $\Lambda$ form factors in the present work and proton form factors in Refs. [5,6,25,27] indicate that the VMD model could provide us a good way to describe the electromagnetic form factors of the baryons. Naturally, we could extend the present estimation to investigate the form factors of $\Sigma$ baryon. It should be noticed that the isospin of $\Sigma$ is one, thus the contributions from $\rho$ meson and its excited states should also be considered.

\section{ACKNOWLEDGMENTS}

This work is partially supported by the NSFC under Grants No. 11575043 and No. 11775050, by the Fundamental Research Funds for the Central Universities of China. Y. Y. is supported by the Scientific Research Foundation of Graduate School of Southeast University (Grant No. YBJJ1770) and the Postgraduate Research \& Practice Innovation Program of Jiangsu Province (Grants No. KYCX17_0043). 
[1] N. Cabibbo and R. Gatto, Pion Form Factors from Possible High-Energy Electron-Positron Experiments, Phys. Rev. Lett. 4, 313 (1960).

[2] N. Cabibbo and R. Gatto, Electron positron colliding beam experiments, Phys. Rev. 124, 1577 (1961).

[3] S. Pacetti, R. Baldini Ferroli, and E. Tomasi-Gustafsson, Proton electromagnetic form factors: Basic notions, present achievements and future perspectives, Phys. Rep. 550-551, 1 (2015).

[4] A. Denig and G. Salme, Nucleon electromagnetic form factors in the timelike region, Prog. Part. Nucl. Phys. 68, 113 (2013).

[5] F. Iachello and Q. Wan, Structure of the nucleon from electromagnetic timelike form factors, Phys. Rev. C 69, 055204 (2004).

[6] R. Bijker, Space- and time electromagnetic form-factors of the nucleon, Rev. Mex. Fis. Suppl. 52, 0117 (2006).

[7] B. Delcourt et al., Study of the reaction $e^{+} e^{-} \rightarrow p \bar{p}$ in the total energy range $1925 \mathrm{MeV}-2180 \mathrm{MeV}$, Phys. Lett. B 86, 395 (1979).

[8] D. Bisello et al. (DM2 Collaboration), Baryon pair production in $e^{+} e^{-}$annihilation at $\sqrt{S}=2.4 \mathrm{GeV}$, Z. Phys. C 48, 23 (1990).

[9] T. A. Armstrong et al. (E760 Collaboration), Measurement of the Proton Electromagnetic Form-Factors in the Timelike Region at $8.9 \mathrm{GeV}^{2}-13 \mathrm{GeV}^{2}$, Phys. Rev. Lett. 70, 1212 (1993).

[10] G. Bardin et al., Determination of the electric and magnetic form-factors of the proton in the timelike region, Nucl. Phys. B411, 3 (1994).

[11] A. Antonelli et al., The first measurement of the neutron electromagnetic form-factors in the timelike region, Nucl. Phys. B517, 3 (1998).

[12] E. A. Kuraev, E. Tomasi-Gustafsson, and A. Dbeyssi, A model for space and time proton (neutron) form factors, Phys. Lett. B 712, 240 (2012).

[13] M. Andreotti et al., Measurements of the magnetic formfactor of the proton for timelike momentum transfers, Phys. Lett. B 559, 20 (2003).

[14] S. J. Brodsky, C. E. Carlson, J. R. Hiller, and D. S. Hwang, Single spin polarization effects and the determination of time-like proton form-factors, Phys. Rev. D 69, 054022 (2004).

[15] B. Aubert et al. (BABAR Collaboration), A study of $e^{+} e^{-} \rightarrow$ $p \bar{p}$ using initial state radiation with BABAR, Phys. Rev. D 73, 012005 (2006).

[16] T. K. Pedlar et al. (CLEO Collaboration), Precision Measurements of the Timelike Electromagnetic FormFactors of Pion, Kaon, and Proton, Phys. Rev. Lett. 95, 261803 (2005).

[17] E. Tomasi-Gustafsson, F. Lacroix, C. Duterte, and G. I. Gakh, Nucleon electromagnetic form-factors and polarization observables in space and time regions, Eur. Phys. J. A 24, 419 (2005).

[18] J. P. Lees et al. (BABAR Collaboration), Study of $e^{+} e^{-} \rightarrow$ $p \bar{p}$ via initial-state radiation at BABAR, Phys. Rev. D 87, 092005 (2013).

[19] J. P. Lees et al. (BABAR Collaboration), Measurement of the $e^{+} e^{-} \rightarrow p \bar{p}$ cross section in the energy range from 3.0 to 6.5 GeV, Phys. Rev. D 88, 072009 (2013).
[20] M. N. Achasov et al., Study of the process $e^{+} e^{-} \rightarrow n \bar{n}$ at the VEPP-2000 $e^{+} e^{-}$collider with the SND detector, Phys. Rev. D 90, 112007 (2014).

[21] J. Haidenbauer, X.-W. Kang, and U.-G. Meißner, The electromagnetic form factors of the proton in the timelike region, Nucl. Phys. A929, 102 (2014).

[22] R. R. Akhmetshin et al. (CMD-3 Collaboration), Study of the process $e^{+} e^{-} \rightarrow p \bar{p}$ in the c.m. energy range from threshold to $2 \mathrm{GeV}$ with the CMD-3 detector, Phys. Lett. B 759, 634 (2016).

[23] M. Ablikim et al. (BESIII Collaboration), Measurement of the proton form factor by studying $e^{+} e^{-} \rightarrow p \bar{p}$, Phys. Rev. D 91, 112004 (2015).

[24] E. L. Lomon, Effect of recent R(p) and R(n) measurements on extended Gari-Krumpelmann model fits to nucleon electromagnetic form-factors, Phys. Rev. C 66, 045501 (2002).

[25] R. Bijker and F. Iachello, Re-analysis of the nucleon spaceand time electromagnetic form-factors in a two-component model, Phys. Rev. C 69, 068201 (2004).

[26] F. Iachello, Structure of the nucleon from electromagnetic form factors, Fiz. B 13, 13 (2004) [Eur. Phys. J. A 19, 29 (2004)].

[27] F. Iachello, A. D. Jackson, and A. Lande, Semiphenomenological fits to nucleon electromagnetic form-factors, Phys. Lett. 43B, 191 (1973).

[28] G. P. Lepage and S. J. Brodsky, Exclusive Processes in Quantum Chromodynamics: The Form-Factors of Baryons at Large Momentum Transfer, Phys. Rev. Lett. 43, 545 (1979); Erratum, Phys. Rev. Lett. 43, 1625(E) (1979).

[29] G. P. Lepage and S. J. Brodsky, Exclusive processes in perturbative quantum chromodynamics, Phys. Rev. D 22, 2157 (1980).

[30] A. V. Belitsky, X. d. Ji, and F. Yuan, A Perturbative QCD Analysis of the Nucleon's Pauli Form-Factor $F_{2}\left(Q^{2}\right)$, Phys. Rev. Lett. 91, 092003 (2003).

[31] J. Haidenbauer and U. G. Meißner, The electromagnetic form factors of the $\Lambda$ in the timelike region, Phys. Lett. B 761, 456 (2016).

[32] J. Haidenbauer, T. Hippchen, K. Holinde, B. Holzenkamp, V. Mull, and J. Speth, The reaction $\bar{p} p \rightarrow \bar{\Lambda} \Lambda$ in the meson exchange picture, Phys. Rev. C 45, 931 (1992).

[33] X. Cao, J.P. Dai, and Y.P. Xie, Vector mesons and electromagnetic form factor of the $\Lambda$ hyperon, Phys. Rev. D 98, 094006 (2018).

[34] Y. Yang and Z. Lu, The electromagnetic form factors of $\Lambda$ hyperon in $e^{+} e^{-} \rightarrow \bar{\Lambda} \Lambda$, Mod. Phys. Lett. A 33, 1850133 (2018).

[35] G. Fäldt and A. Kupsc, Hadronic structure functions in the $e^{+} e^{-} \rightarrow \bar{\Lambda} \Lambda$ reaction, Phys. Lett. B 772, 16 (2017).

[36] G. Fäldt, Polarization observables in the $e^{+} e^{-} \rightarrow \bar{\Lambda} \Lambda$ reaction, Eur. Phys. J. A 52, 141 (2016).

[37] O. D. Dalkarov, P. A. Khakhulin, and A. Y. Voronin, On the electromagnetic form factors of hadrons in the time region near threshold, Nucl. Phys. A833, 104 (2010).

[38] R. Baldini, S. Pacetti, A. Zallo, and A. Zichichi, Unexpected features of $e^{+} e^{-} \rightarrow p \bar{p}$ and $e^{+} e^{-} \rightarrow \Lambda \bar{\Lambda}$ cross sections near threshold, Eur. Phys. J. A 39, 315 (2009).

[39] B. Aubert et al. (BABAR Collaboration), Study of $e^{+} e^{-} \rightarrow \Lambda \bar{\Lambda}, \Lambda \bar{\Sigma}^{0}, \Sigma^{0} \bar{\Sigma}^{0}$ using initial state radiation with BABAR, Phys. Rev. D 76, 092006 (2007). 
[40] M. Ablikim et al. (BESIII Collaboration), Observation of a cross-section enhancement near mass threshold in $e^{+} e^{-} \rightarrow$ $\Lambda \bar{\Lambda}$, Phys. Rev. D 97, 032013 (2018).

[41] S. Dobbs, A. Tomaradze, T. Xiao, K. K. Seth, and G. Bonvicini, First measurements of timelike form factors of the hyperons, $\Lambda^{0}, \Sigma^{0}, \Sigma^{+}, \Xi^{0}, \Xi^{-}$, and $\Omega$, and evidence of diquark correlations, Phys. Lett. B 739, 90 (2014).

[42] M. Ablikim et al. (BESIII Collaboration), Study of $J / \psi$ and $\psi(3686)$ decay to $\Lambda \bar{\Lambda}$ and $\Sigma^{0} \bar{\Sigma}^{0}$ final states, Phys. Rev. D 95, 052003 (2017).

[43] I. T. Lorenz, H. W. Hammer, and U. G. Meißner, New structures in the proton-antiproton system, Phys. Rev. D 92, 034018 (2015).

[44] P. Lichard, Common explanation of the behavior of some $e^{+} e^{-}$annihilation processes around $\sqrt{s}=1.9 \mathrm{GeV}$, Phys. Rev. D 98, 113011 (2018).

[45] M. Ablikim et al. (BESIII Collaboration), Complete Measurement of $\Lambda$ Electromagnetic form Factors, Phys. Rev. Lett. 123, 122003 (2019).

[46] S. J. Brodsky, J. R. Hiller, D. S. Hwang, and V. A. Karmanov, The covariant structure of light front wave functions and the behavior of hadronic form-factors, Phys. Rev. D 69, 076001 (2004).

[47] M. Tanabashi et al. (Particle Data Group), Review of particle physics, Phys. Rev. D 98, 030001 (2018).

[48] Y. B. Dong, A. Faessler, and K. Shimizu, Nucleon formfactors in a relativistic quark model, Eur. Phys. J. A 6, 203 (1999).

[49] F. Huang, R. G. Xu, and B. Q. Ma, Sigma meson cloud and proton's light flavor sea quarks, Phys. Lett. B 602, 67 (2004).

[50] D. Y. Chen, Y. B. Dong, M. M. Giannini, and E. Santopinto, Hypercentral constituent quark model with a meson cloud, Nucl. Phys. A782, 62 (2007).

[51] D. Y. Chen and Y. B. Dong, Nucleon form factors and $N-\Delta$ transitions in a hypercentral constituent quark model with meson cloud, Commun. Theor. Phys. 47, 539 (2007).
[52] A. B. Arbuzov and T. V. Kopylova, On relativization of the Sommerfeld-Gamow-Sakharov factor, J. High Energy Phys. 04 (2012) 009.

[53] W. R. Frazer and J. R. Fulco, Effect of a pion-pion scattering resonance on nucleon structure. II, Phys. Rev. 117, 1609 (1960).

[54] H. B. O'Connell, B. C. Pearce, A. W. Thomas, and A. G. Williams, $\rho-\omega$ mixing, vector meson dominance and the pion form-factor, Prog. Part. Nucl. Phys. 39, 201 (1997).

[55] J. P. Lees et al. (BABAR Collaboration), Precise measurement of the $e^{+} e^{-} \rightarrow \pi^{+} \pi^{-}(\gamma)$ cross section with the initialstate radiation method at BABAR, Phys. Rev. D 86, 032013 (2012).

[56] J. P. Lees et al. (BABAR Collaboration), Cross sections for the reactions $e^{+} e^{-} \rightarrow K_{S}^{0} K_{L}^{0}, K_{S}^{0} K_{L}^{0} \pi^{+} \pi^{-}, K_{S}^{0} K_{S}^{0} \pi^{+} \pi^{-}$, and $K_{S}^{0} K_{S}^{0} K^{+} K^{-}$from events with initial-state radiation, Phys. Rev. D 89, 092002 (2014).

[57] R. R. Akhmetshin et al. (CMD-3 Collaboration), Study of the process $e^{+} e^{-} \rightarrow \pi^{+} \pi^{-} \pi^{0} \eta$ in the c.m. energy range 1394-2005 MeV with the CMD-3 detector, Phys. Lett. B 773, 150 (2017).

[58] M. Ablikim et al. (BESIII Collaboration), Study of $J / \psi \rightarrow$ $\eta \phi \pi^{+} \pi^{-}$at BESIII, Phys. Rev. D 91, 052017 (2015).

[59] H. Sanchis-Alepuz and C.S. Fischer, Hyperon elastic electromagnetic form factors in the space-like momentum region, Eur. Phys. J. A 52, 34 (2016).

[60] G. Ramalho, K. Tsushima, and A. W. Thomas, Octet Baryon electromagnetic form factors in nuclear medium, J. Phys. G 40, 015102 (2013).

[61] S. Boinepalli, D. B. Leinweber, A. G. Williams, J. M. Zanotti, and J. B. Zhang, Precision electromagnetic structure of octet baryons in the chiral regime, Phys. Rev. D 74, 093005 (2006).

[62] G. Wagner, A. J. Buchmann, and A. Faessler, Magnetic moments of the octet baryons in a chiral quark potential model, Phys. Lett. B 359, 288 (1995).

[63] G. Wagner, A. J. Buchmann, and A. Faessler, Exchange currents in octet hyperon charge radii, Phys. Rev. C 58, 3666 (1998). 